\title{
Dealing with seasonal pseudohypokalemia in paediatric patients due to high ambient temperature in tropical climate: A laboratory perspective
}

\author{
Sucharita Mohanty ${ }^{1}$, Alpana Mishra ${ }^{2, *}$ \\ ${ }^{\mathbf{1}}$ Associate Professor, Dept. of Biochemistry, MKCG Medical College and Hospital, Berhampur, Odisha, ${ }^{\mathbf{2}}$ Assistant Professor, \\ Dept. of Community Medicine, KIMS, Bhubaneswar, Odisha, India \\ *Corresponding Author: \\ Email: dr.alpanamishraspm@gmail.com
}

Received: $18^{\text {th }}$ February, 2018

Accepted: $14^{\text {th }}$ April, 2018

\begin{abstract}
Introduction: During summer 2015, we had observed a significant rise in incidence of hypokalemia in comparison to winter 2014-2015. We speculated these findings might have occurred as a result of delay in centrifugation during which the samples were exposed to the high ambient temperature. So we took up the study to find out whether reducing the time period between venipuncture and centrifugation can be of any help to reduce the magnitude of incidence of pseudohypokalemia during summer months.

Materials and Methods: We reduced the time between venipuncture and serum separation to less than thirty minutes for all the samples received at the laboratory in the year 2015-2016.

Estimation of serum potassium was done within thirty minutes of serum separation.

Result: In the year 2014- 2015 the difference between proportion of hypokalemia in summer and winter was statistically significant $(\mathrm{p}<0.0001)$ whereas the difference in proportion of hypokalemia in 2015-2016 between summer and winter was not statistically significant $(\mathrm{p}<0.897)$.

Conclusion: The laboratory people should be aware of the fact that delays in sample centrifugation and estimation of serum potassium results in spuriously low serum potassium value at high ambient temperature. Reducing the time of serum separation and estimation of serum potassium to within one hour can solve the problem of pseudohypokalemia and thereby improve the patient management.
\end{abstract}

Keywords: Seasonal pseudohypokalemia, Tropical climate, High ambient temperature, Paediatric patients.

\section{Introduction}

Pre-analytical phase is the most vulnerable part of the total testing process and is considered to be among the greatest challenges to the laboratory professionals. ${ }^{1}$ For some of the most critical procedures within the preanalytical phase internationally accepted guidelines and recommendations as well as related quality control measures are unfortunately unavailable. ${ }^{2}$ The majority of errors (32-75\%) occur before the sample is analyzed: during labeling, collection, transport or centrifugation. ${ }^{3}$ It has been estimated that $60-70 \%$ of clinical decisions are based on laboratory results and potassium is among the ten most commonly tested analytes. ${ }^{4}$ Spurious hypoand hyperkalemia have been a problem in the laboratory ever since serum and plasma potassium was first measured. Studies have shown a significant inverse correlation between daily temperature and mean potassium concentration..$^{5-9}$ In our previous study, we observed that high ambient temperature is a cause of spuriously low serum potassium concentration in paediatric patients when there is a delay in time between venipuncture and separation of serum by centrifugation by more than one hour. ${ }^{10}$ Spurious laboratory results are results that are analytically correct but do not accurately reflect in vivo plasma analyte concentrations. Spurious electrolyte results often lead to unnecessary testing or injudicious treatment and have adverse effect on patient outcome. ${ }^{11}$
Under these circumstances, we took up the study to find out whether shortening the time between venipuncture and serum separation can be of any help to abate seasonal pseudohypokalemia due to high ambient temperature.

\section{Materials and Methods}

The indoor paediatric patients of SVP Post Graduate Institute of Paediatrics, Cuttack, Odisha, in the age group of 1-14 years were selected as the study group. The duration of study was from December to June for the year $2014-2015$ and for the same time frame in 2015 - 2016. To study the seasonal variation in serum potassium level, the study data was categorized into summer and winter seasons: winter season (December-February) and summer (MarchJune).

In the year 2014 - 2015, the time between venipuncture and serum separation by centrifugation was more than one hour and during that period the samples were exposed to the ambient temperature of that day. In the year $2014-2015$, no strict protocol was followed regarding the timing of centrifugation and analysis of serum potassium thereafter. To find out the incidence of hypokalemia a retrospective record based data for serum potassium was collected excluding the cases having high serum urea, creatinine, altered $\mathrm{Na}^{+}$ and sugar values. 
The time between venipuncture and serum separation by centrifugation was reduced to less than thirty minutes in the year 2015 - 2016 as a protocol, assuming it to be a major pre-analytical variable causing seasonal pseudohypokalemia. The staffs in different wards were instructed to mention the time of venipuncture whenever a blood was to be drawn for any analysis and to send the sample to the laboratory without delay. The laboratory staffs were instructed to centrifuge the samples within 30 minutes from the time of venipuncture and record the time of centrifugation of the samples. The sample collection and centrifugation room was not air-conditioned during the study period. The centrifuged samples were then transferred to the laboratory where the samples were processed. The temperature inside the laboratory was maintained between $22-25^{\circ} \mathrm{C}$. The serum potassium values for the samples having normal urea, creatinine, sugar, $\mathrm{Na}^{+}$ values were included in the study.

After centrifugation, the samples were processed within 30 minutes. This practice was followed in the laboratory for all the routine biochemical tests. The estimation of serum potassium was done one by one and it took one and half minute for the assay to be finished.

The serum potassium level was measured in HDC electrolyte analyzer using ion selective electrode. The internal quality assurance was done by the control provided by the manufacturer. External quality assurance was done with the control provided by BioRad. The performance of the analyzer over the years was satisfactory according to the results of both internal and external quality control measures.

The samples with serum potassium value $<3.5$ $\mathrm{mmol} / \mathrm{L}$ were considered as hypokalemic.

The daily maximum temperature was recorded as per the data from regional meteorological department.

The statistical analysis was done using MS Excel, SPSS version 21.

\section{Result}

The total number of sample analyzed in the year $2014-2015$ was 3260. The total number of samples analyzed in the year $2015-2016$ was 2708 .
In the year 2014-2015, the mean temperature during winter was $29.79 \pm 3.16{ }^{\circ} \mathrm{C}$ and during summer was $37.29 \pm 2.91{ }^{0} \mathrm{C}$. The difference in mean temperature in winter and summer was statistically significant $(\mathrm{p}<0.0001)$. The mean serum potassium during winter was $4.53 \pm 0.89 \mathrm{mmol} / \mathrm{L}$ and mean serum potassium during summer was $4.297 \pm 0.929 \mathrm{mmol} / \mathrm{L}$. The difference in mean serum potassium level during summer and winter was statistically significant $(\mathrm{p}<0.0001)$. (Table 1)

The mean temperature during winter was $31.52 \pm$ $2.91^{\circ} \mathrm{C}$ and during summer was $38.08 \pm 3.37^{\circ} \mathrm{C}$ in the year 2015-2016. The difference in mean temperature in winter and summer was statistically significant $(\mathrm{p}<0.0001)$. The mean serum potassium during winter was $4.396 \pm 0.746 \mathrm{mmol} / \mathrm{L}$ and mean serum potassium during summer was $4.429 \pm 0.772 \mathrm{mmol} / \mathrm{L}$. The difference in mean serum potassium level during summer and winter was not significant statistically ( $\mathrm{p}=$ 0.260 ). (Table 2) The mean time of centrifugation in winter was $24.35 \pm 4.0$ minutes and summer it was $24.29 \pm 3.953$ minutes. There was no significant difference in timing of centrifugation between both the groups $(\mathrm{t}=0.434, \mathrm{p}=0.665)$.

Out of 1611 samples analyzed in winter (20142015), 170 samples (10.6\%) were hypokalemic. Whereas in summer (2014-2015) out of 1649 samples 279 samples (16.9\%) were hypokalemic (Table 3). The difference between proportion of hypokalemia in summer and winter was statistically significant $(\mathrm{p}<0.0001)$. The chance of getting a hypokalemic sample in summer was 1.7 times higher as compared to winter, which was also statistically significant.

In the year 2015-2016, out of 1207 samples analyzed 134 samples $(11.1 \%)$ were hypokalemic during winter season. In summer (2015-2016), 169 samples $(11.3 \%)$ were hypokalemic out of 1501 samples (Table 4). The difference between proportion of hypokalemia in summer and winter was not statistically significant $(\mathrm{p}<0.897)$.

Table 1: Seasonal distribution of temperature andserum potassium level in the year 2014 -2015

\begin{tabular}{|l|c|c|c|c|}
\hline & Winter & Summer & t value & p value \\
\hline Temperature in ${ }^{0} \mathrm{c}$ & $29.79 \pm 3.16$ & $37.29 \pm 2.91$ & -70.485 & $<0.0001$ \\
\hline Potassium in $\mathrm{mmol} / \mathrm{L}$ & $4.53 \pm 0.89$ & $4.297 \pm 0.929$ & 7.472 & $<0.0001$ \\
\hline
\end{tabular}

Table 2: Seasonal distribution of temperature and serum potassium level in the year 2015 -2016 with time of centrifugation

\begin{tabular}{|l|c|c|c|c|}
\hline & Winter & Summer & t Value & p Value \\
\hline Time of centrifugation & $24.35 \pm 4.0$ & $24.29 \pm 3.95$ & 0.434 & $\mathrm{p}=0.665$ \\
\hline Temperature in ${ }^{0} \mathrm{c}$ & $31.52 \pm 2.91$ & $38.08 \pm 3.37$ & -53.41 & $<0.0001$ \\
\hline Potassium in $\mathrm{mmol} / \mathrm{L}$ & $4.396 \pm 0.746$ & $4.429 \pm 0.772$ & -1.127 & 0.260 \\
\hline
\end{tabular}


Table 3: Proportion of hypokalemic samples with delayed centrifugation in the year 2014 -2015

\begin{tabular}{|l|c|c|}
\hline & Winter & Summer \\
\hline Total number of samples & 1611 & 1649 \\
\hline Number of hypokalemic samples & 170 & 279 \\
\hline $\begin{array}{l}\text { Percentage of hypokalemic } \\
\text { samples }\end{array}$ & 10.6 & 16.9 \\
\hline $\begin{array}{l}\text { Chi } \mathrm{q}=27.815, \mathrm{df}=1, \mathrm{p}<0.0001 \\
\text { Odds Ratio= } 1.726(1.407-2.118)\end{array}$ & \\
\hline
\end{tabular}

Table 4: Proportion of hypokalemic samples with early centrifugation in the year 2015 -2016

\begin{tabular}{|l|c|c|}
\hline & Winter & Summer \\
\hline Total number of samples & 1207 & 1501 \\
\hline Number of hypokalemic samples & 134 & 169 \\
\hline $\begin{array}{l}\text { Percentage (\%)of hypokalemic } \\
\text { samples }\end{array}$ & 11.1 & 11.3 \\
\hline $\begin{array}{l}\text { Chi sq }=0.017, \mathrm{df}=1, \mathrm{p}=0.897 \\
\text { Odds Ratio= } 1.016(0.799-1.292)\end{array}$ & \\
\hline
\end{tabular}

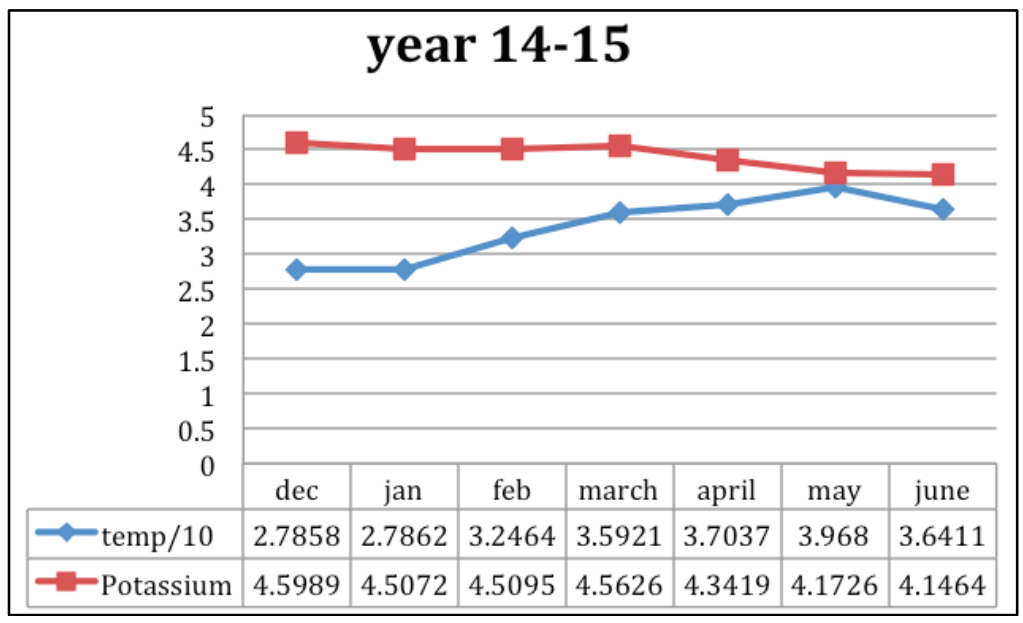

Fig. 1: Line graph of monthly mean serum potassium with monthly mean temperature in degree Celsius/ 10 for the year 2014-2015

Fig. 1 shows the relation between mean serum potassium in $\mathrm{mmol} / \mathrm{L}$ and temperature in degree Celsius/10 for the year 2014-2015. A negative correlation was observed between mean potassium values and mean temperatures during both the seasons.

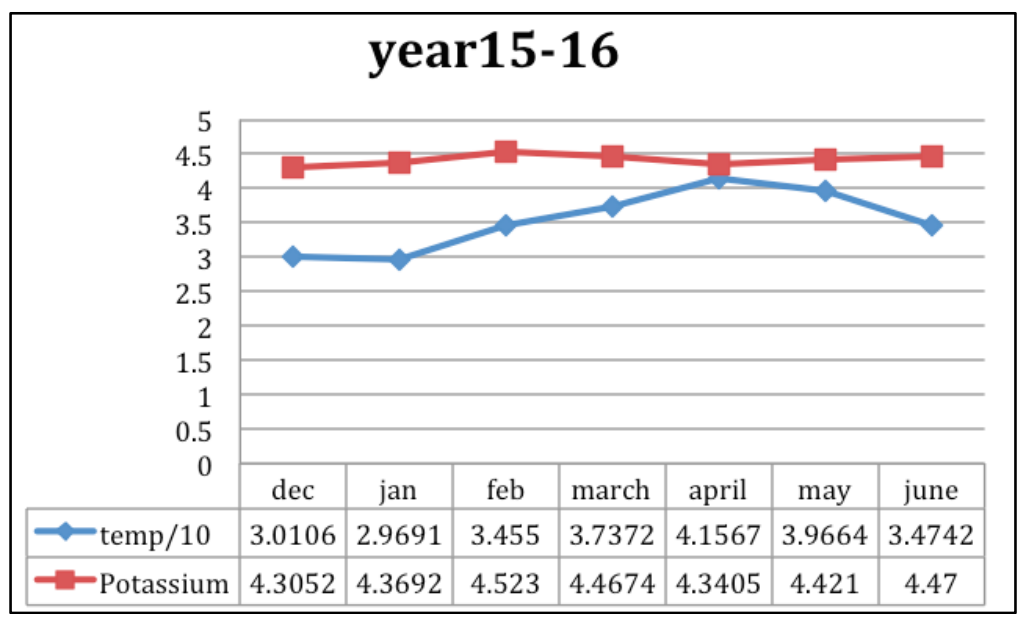

Fig. 2: Line graph of monthly mean serum potassium with monthly mean temperature in degree Celsius/10 for the year 2015-2016 
Fig. 2 shows the relation between mean serum potassium in $\mathrm{mmol} / \mathrm{L}$ and temperature in degree Celsius/10 for the year 2015-2016. A negative correlation was observed between mean potassium values and mean temperatures during both the seasons. Pearson correlation coefficient was 0.044 and $\mathrm{p}=0.023$ which was not significant statistically.

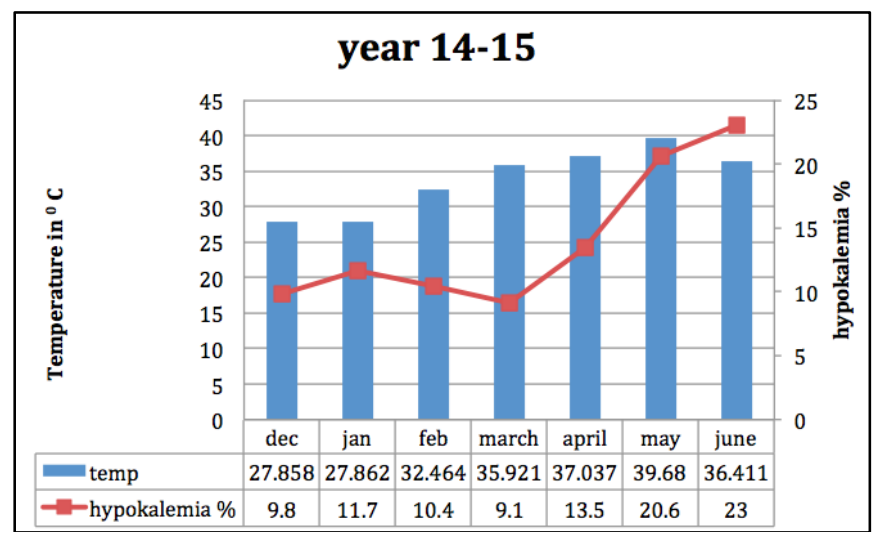

Fig. 3: Monthly distribution of percentage of hypokalemia with mean monthly temperature for the year 20142015 (Before reducing the time between venipuncture and centrifugation)

Fig. 3 shows the percentage of hypokalemia with mean temperature on monthly basis for the year 2014-2015. There was a rise in percentage of hypokalemic cases with the rise of temperature during summer season.

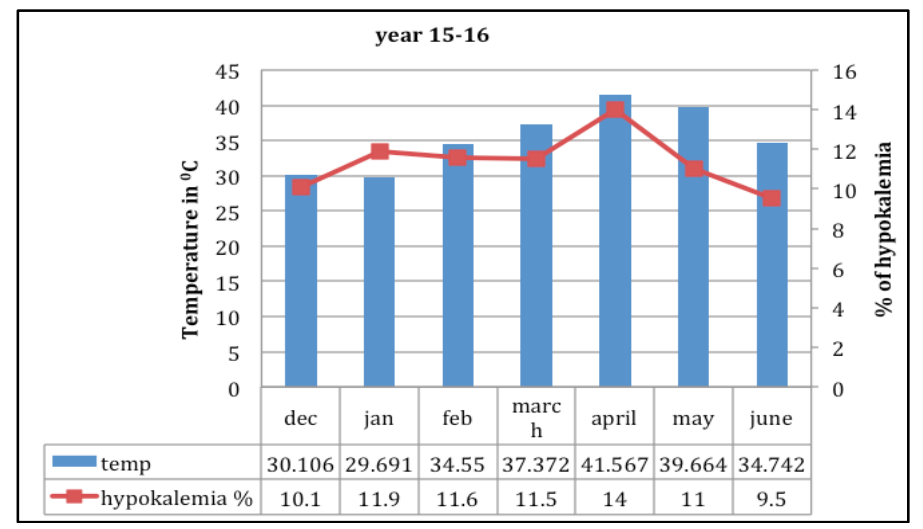

Fig. 4: Monthly distribution of percentage of hypokalemia with mean monthly temperature for the year 20152016 (After reducing the time between venipuncture and centrifugation)

Fig. 4 shows the percentage of hypokalemia with mean temperature on monthly basis for the year 2015-2016.

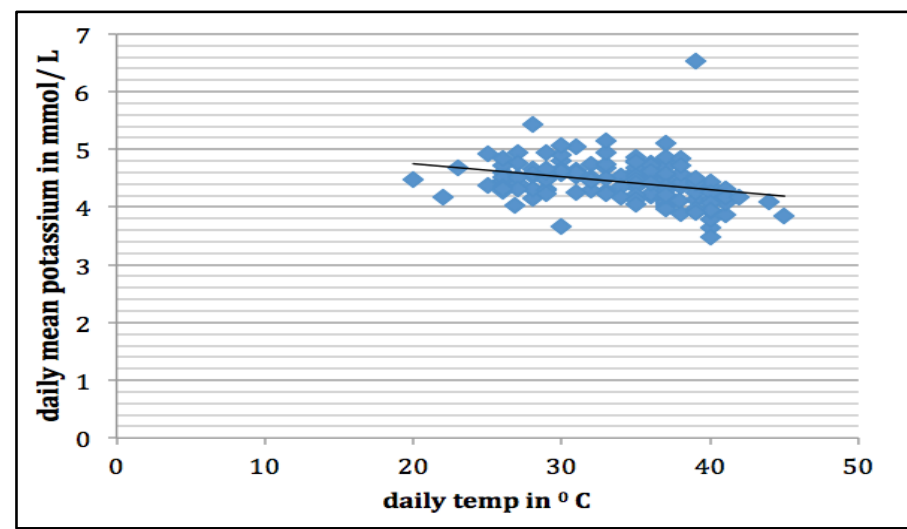

Fig. 5: Scatter plots of daily temperature and daily mean potassium for the year 2014-2015 
Fig. 5 Scatter diagram showing variation of daily mean serum potassium concentration with daily mean temperature for the year 2014-2015. Pearson correlation coefficient was-0.311 and $\mathrm{p}<0.0001$ which was statistically significant.

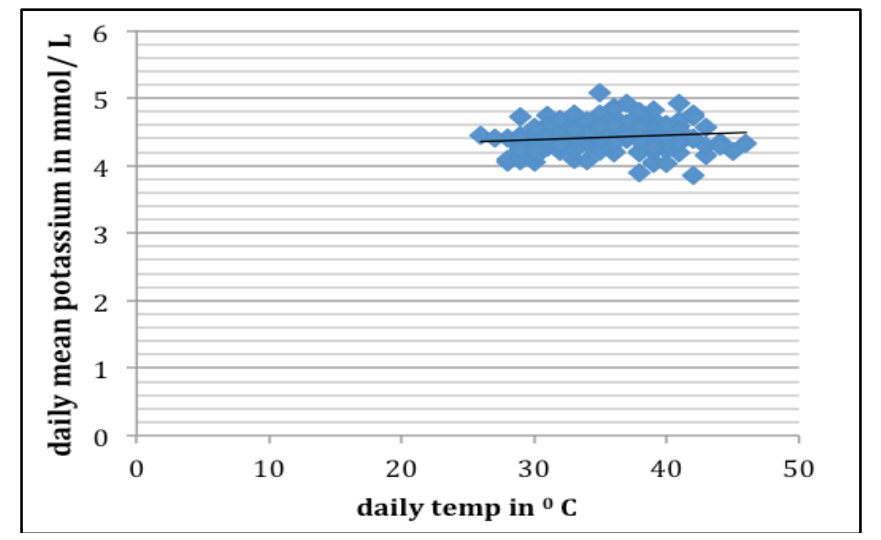

Fig. 6: Scatter plots of daily temperature and daily mean potassium for the year 2015-2016

Fig. 6 Scatter diagram showing variation of daily mean serum potassium concentration with daily mean temperature for the year 2015-2016. Pearson correlation coefficient was 0.141 and $p=0.082$ which was not statistically significant.

\section{Discussion}

The month of December, January and February were taken as winter season and March, April, May and June as summer season according to the monthly mean temperature of our locality. To negate the variation of ambient temperature we have chosen the same time periods as winter and summer for both the years of our study.

A significant rise $(p<0.0001)$ in incidence of pseudohypokalemia was observed in summer season in comparison to winter, when there was a delay in centrifugation of the samples for more than one hour during which the samples were exposed to high ambient temperature of that day in 2014-2015. Our finding is consistent with other studies, which have reported the phenomenon of seasonal pseudohypokalemia, which is contributed by prolonged exposure to higher than average temperature ${ }^{5-9}$ A negative correlation between ambient temperature and incidence of hypokalemia, was observed when there was a delay in centrifugation. There was a significant negative correlation between mean monthly temperature and mean monthly potassium. Other studies have also found a trend towards lower mean daily potassium concentrations at higher ambient temperatures. ${ }^{5,6,12}$

The mean serum potassium value in summer 2015 was significantly lower $(\mathrm{p}<0.0001)$ than that in winter (2014-15), which is in agreement with other investigators. ${ }^{6}$ Some investigators observed an initial fall of serum potassium up to $-0.22 \mathrm{mmol} / \mathrm{L}$ when samples were kept at $37^{\circ} \mathrm{C}$ for 4 hours. ${ }^{5}$ A $10^{\circ} \mathrm{C}$ rise in temperature, which is possible even in temperate climates, could produce up to $0.2 \mathrm{mmol} / \mathrm{L}$ decrease in potassium concentration when there is delay between sample collection and analysis. ${ }^{6}$
At higher temperature there is a higher metabolic rate with an increased potassium uptake. ${ }^{13}$ It is well known that $\mathrm{Na}^{+}-\mathrm{K}^{+}$exchanging ATPase activity is enhanced by increased temperature.

When we reduced the time between venipuncture and serum separation by centrifugation to less than 30 minutes we did not notice any significant difference $(\mathrm{p}=0.897)$ in incidence of pseudohypokalemia between summer and winter season 2015-2016, although there was a significant difference $(\mathrm{p}<0.0001)$ in temperatures between both the seasons. Our study is in agreement with studies that have suggested for early centrifugation of sample for estimation of serum potassium level. ${ }^{12,14}$ Ideally blood samples for measurement of potassium should be separated within one hour of venipuncture. The longer the plasma or serum is left in contact with the blood cells, the less likely the measured potassium will reflect the true in vivo concentration. ${ }^{7}$

The proportion of hypokalemia in summer 2016 $(11.3 \%)$ was significantly lower $(p<0.001)$ than in summer $2015(16.9 \%)$. Whereas no significant difference $(\mathrm{p}=0.7912)$ in the proportion of hypokalemia was observed between winter 2014-2015 (10.6\%) and winter 2015-2016 (11.1\%). Other investigators have predicted a rise in proportion of un-diseased population with a low potassium could rise from $2.5 \%$ to $15 \%$ in very hot weather. ${ }^{12}$ In a tropical country like India the temperature varied from $26^{\circ} \mathrm{C}$ to $34.5^{\circ} \mathrm{C}$ even in winter, which may be the cause of increased proportion of hypokalemia observed in our study population.

The mean serum potassium level showed no significant difference $(\mathrm{p}=0.260)$ in the year 2015-2016 winter and summer although it was highly significant $(p<0.0001)$ in the year 2014-2015. This may be due to reduction in time interval between sample collection and analysis, which has pushed the value towards normokalemia, thus reducing the difference in mean 
serum potassium values between the seasons.

In 2015-2016 the blood samples were centrifuged within 30 minutes of collection and the serum potassium level was analyzed within 30 minutes of centrifugation. The time between venipuncture and analysis of serum potassium was well within one hour in 2015-2016. Some investigators have suggested that in tropical country like India the samples for serum electrolyte should be measured as soon as they are received preferably within 1-2 hours. ${ }^{15}$ Our study confirms that the proportion of hypokalemic samples remain fairly constant throughout the year when the time interval between sample collection, serum separation and serum potassium estimation is reduced to within one hour. Ideally blood samples for measurement of potassium should be separated within one hour of venipuncture. The longer the plasma or serum is left with the blood cells, the less likely the measured potassium will reflect the true in vivo concentration. ${ }^{7}$

Laboratory testing is divided into three phases- preanalytical, analytical and post analytical. Most errors occur in the pre-analytical phase $(48-68.2 \%) .{ }^{16}$ Each laboratory result is only as good as the specimen from whom the value is derived. The way a blood specimen is handled and processed in the period of time after collection to just before analysis is critically important to the process of ensuring accurate, precise, and valid results, which are often used to make critical decisions that can greatly impact patient care. ${ }^{17}$ Reducing the time taken for samples to reach the clinical laboratory may be the most practical way to reduce pseudohypokalemia due to high ambient temperature. In tropical countries laboratories should strive to reduce turn around time to within an hour for serum potassium estimation in order to get a true in vivo potassium level. However further research is needed to find out if similar situation is seen in normal children also.

\section{Conclusion}

In a tropical country like India where the temperature varies from $26^{\circ} \mathrm{C}$ to $45^{\circ} \mathrm{C}$ in summer, the measured value of serum potassium is often spuriously low which poses problem to both clinicians and laboratory personnel. Repeated sampling in paediatric age group should be avoided as it causes inconvenience to the child. If the (spuriously) low serum potassium value is taken into account, the clinicians may initiate treatment that may not be appropriate. To avoid the inconveniences, the serum should be separated within thirty minutes of venipuncture and processed within thirty minutes after centrifugation. A local reference range must be made for each laboratory after observing stringent time frame for sample collection, serum separation and estimation of serum potassium. This will help in appropriate diagnosis and treatment.

\section{Competing Interest: None}

\section{Funding: None}

\section{References}

1. Ana-Maria Simundic, Giuseppe Lippi. Preanalytical phase - a continuous challenge for laboratory professionals. Biochemica Medica. 2012;22(2):145-9.

2. Plebani M, Sciacovelli L, Lippi G. Quality indicators for laboratory diagnostics: consensus is needed. Ann Clin Biochem. 2011;48:479.

3. Stankovik AK, Smith S. Elevated serum potassium values: The role of preanalytic variables. Am J Clin Pathol. 2004;121:S105-12.

4. Forsman RW. Why is the laboratory an afterthought for managed care organizations? Clin Chem.1996;42:813-6.

5. Masters PW, Lawson N, Marenah CB, Maile LJ. High ambient temperature: a spurious cause of hypokalemia. BMJ. 1996;312:1653-9.

6. Ulahannan Tj, MC Vittie J, Kennan J. Ambient temperature and potassium concentration. Lancet. 1998;352:1680-1.

7. Sinclair D, Briston P, Young R, Pepin N. Seasonal pseudohyperkalemia. J Clin Pathol. 2003;56:385-8.

8. Sodi R, Davison AS, Holmes E, Hine TJ, Roberts NB. The phenomenon of seasonal pseudohypokalemia: effect of ambient temperature, plasma glucose and role of sodium-potassium-exchanging ATPase. Clin Biochem 2009;42:813-8.

9. Katie LJ, Soha Z. High ambient temperature and hypokalemia. Annals of Clin Biochem; Jan 2014;51(1) 114-5.

10. Mohanty S, Mishra A. seasonal pseudohypokalemia in padiatric patients in tropical climate. International $J$ of Clin Biochem and Research. 2015,2(4):223-25.

11. RA Sulaiman, PJ Towmey, R Gama. Mitigation and detection of spurious potassium and sodium results. Clinica Chimica Acta. Jan 2011;412(1-2):1-6.

12. Seamark D, Backhouse s, Barber P, Hichens J, Salzmann M, Powell R Transport and temperature effects on measurement of serum and plasma potassium. $J R S O c$ Med.1999;92:339-41.

13. Melissa T, Neil K, Brian S, Stephen H, Michelle L. Stability of common Biochemical analytes in serum gel tubes subjected to various storage temperature and times pre-centrifugation. Ann Clin Biochem. 2008;45:375-79.

14. Masters PW, Ismail AA. Minimizing factitious hyperkalemia: samples should be centrifuged after collection in general practices. BMJ. 1997;315:190.

15. Ankur B, Parul G, Saket S, Ramesh K L, Rashmirashi D. Delay in Specimen Processing-Major Source of Preanalytical Variation in Serum Electrolytes. Journal of Clinical and Diagnostic Research. 2014;8(12):1-3.

16. Phlebani M, Errors in clinical laboratory or errors in laboratory medicine? Clin Chem Lab Med. 2006;44(6):750-59.

17. Glen McDaniel. Newly Revised CLSI Guideline Addresses Handling and Processing of Blood Specimens. Lab Med. (2010) 41 (11):694-695.

How to cite this article: Mohanty S, Mishra A.
Dealing with seasonal pseudohypokalemia in
paediatric patients due to high ambient temperature in
tropical climate: A laboratory perspective. Int J Clin
Biochem Res. $2018 ; 5(3): 477-482$.

